EPJ Web of Conferences 111,06002 (2016)

DOI: $10.1051 /$ epjconf/201611106002

(C) Owned by the authors, published by EDP Sciences, 2016

\title{
Development of an automated platform for the verification, test- ing, processing and benchmarking of Evaluated Nuclear Data at the NEA Data Bank
}

\section{Status of the NDEC system}

\author{
F. Michel-Sendis ${ }^{1, a}$, C.J. Díez ${ }^{1}$, and O. Cabellos ${ }^{1}$ \\ ${ }^{1}$ OECD Nuclear Energy Agency (NEA) - Data Bank, 92130, Issy-les-Moulineaux, France
}

\begin{abstract}
Modern nuclear data Quality Assurance (QA) is, in practice, a multistage process that aims at establishing a thorough assessment of the validity of the physical information contained in an evaluated nuclear data file as compared to our best knowledge of available experimental data and theoretical models. It should systematically address the performance of the evaluated file against available pertinent integral experiments, with proper and prior verification that the information encoded in the evaluation is accurately processed and reconstructed for the application conditions. The aim of the NDEC (Nuclear Data Evaluation Cycle) platform currently being developed by the Data Bank is to provide a correct and automated handling of these diverse QA steps in order to facilitate the expert human assessment of evaluated nuclear data files, both by the evaluators and by the end users of nuclear data.
\end{abstract}

\section{Introduction}

Quality Assurance (QA) applied to nuclear data is designed to ensure that a comprehensive set of quality requirements are fulfilled for electronic evaluated nuclear data files. These requirements go well beyond the necessary verification of compliance with the ENDF- 6 format for the electronic files. The verification that the information encoded in the evaluation is accurately processed and reconstructed for the application conditions (e.g. energy grouping and application temperatures) is necessary. Giving a systematic assessment of the performance of each file in pertinent (i.e. sensitive to the nuclide and reactions being tested) internationally evaluated integral benchmark experiments is necessary.

The Data Bank is currently developing an automated platform called NDEC (Nuclear Data Evaluation Cycle) that centralizes such QA processes in an user friendly approach. This paper aims to briefly present the current status of this development.

\section{Development of the NDEC platform}

In practice, evaluated nuclear data QA is a multistage process that implies having the appropriate computational framework and expertise to run different codes and corresponding input sequences,

\footnotetext{
a e-mail: franco.michel-sendis@oecd.org
} 
with the capability to interpret the resulting output and handle the large numbers of secondary files generated per evaluated file tested. The problem of modern Evaluated Nuclear Data QA is an example where centralization and automation of sequenced processes become necessary in order to facilitate a thorough diagnose of the data. Such diagnoses aim at providing both evaluators and end-users of nuclear data with an in-depth understanding of the data's quality, domain of applicability and, importantly, its limitations. This information is essential when selecting candidate evaluations for a reference Nuclear Data Library.

\subsection{Current Status}

The Data Bank started in early 2015 to develop a platform for the systematization of a number of these QA processes, called NDEC (Nuclear Data Evaluation Cycle). Currently, NDEC is an operational prototype that automatically performs a number of steps following the submission of an ENDF-6 evaluated file. These steps will be described below. The longer aim of NDEC is to be available as an online NEA Data Bank service providing access to the output and diagnoses of these different QA steps. Currently the steps handled by NDEC are: evaluated format checking, processing, differential comparisons and integral benchmarking. The resulting output is organized in a user-friendly interface. The codes and tools presently used are briefly described below.

\subsubsection{Evaluated format checking and processing of ENDF-6 files}

ENDF-6 format compliance checks are performed with the utility codes CHECKR, FIZCON, PSYCHE, INTER. Their outputs are compiled. Processing of the submitted file is currently performed with NJOY-2012, PREPRO-2015 and AMPX-6.2. The use of the latter is being carried out by the Data Bank in collaboration with ORNL/SCALE development team under a beta-tester agreement, pending the official release of AMPX (see these proceedings on the processing of JEFF-3.2 with AMPX [1]).

As a general principle, NDEC provides access to all associated inputs used and the outputs obtained by constructing a modular XML organization accessible from a webpage, with modules that are visually color-coded according to the errors or warnings received (see Figure 1) in each sequence performed. A 'dictionary of errors' aims at directly providing a human readable description of the error found in order to facilitate the diagnostic.

\subsubsection{Visualization of differential data}

The user may visualize the comparison of the reconstructed cross-sections with the different processing codes, and includes the comparison to existing EXFOR data and major nuclear data libraries,for each reaction channel. For this NDEC integrates the functionalities of JANIS-4.0 [2] (see Figure 2).

\subsubsection{Integral benchmarking}

Assessing the integral performance of each evaluated nuclear data file in benchmark calculations is an essential step in the QA process, and an important criterion to consider when selecting candidate evaluations for a nuclear data library. Special care should be first given to the selection of benchmarks to run, which depend on the nuclide evaluation / reaction channel / energy domain and, in general, the application being tested for. In order to develop the relevant 'validation suites', the use of the different tools developed by the NEA for these purposes is foreseen. Currently in NDEC, this step is focusing exclusively in criticality benchmarking, with the aims to expand this scope in the future. 


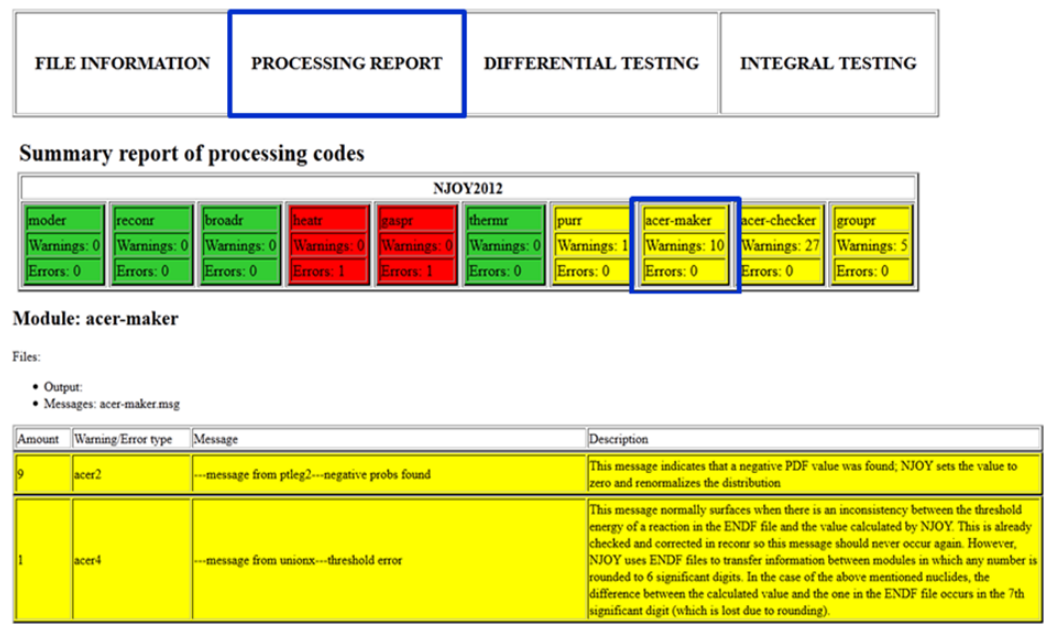

Figure 1. Screen-shot of the 'processing' panel of the NDEC prototype highlighting issues found during NJOY2012 processing, modules are color-coded (green: no errors; yellow: only warnings; red: errors found) and clickable with respective detail of errors and explanations of errors given.
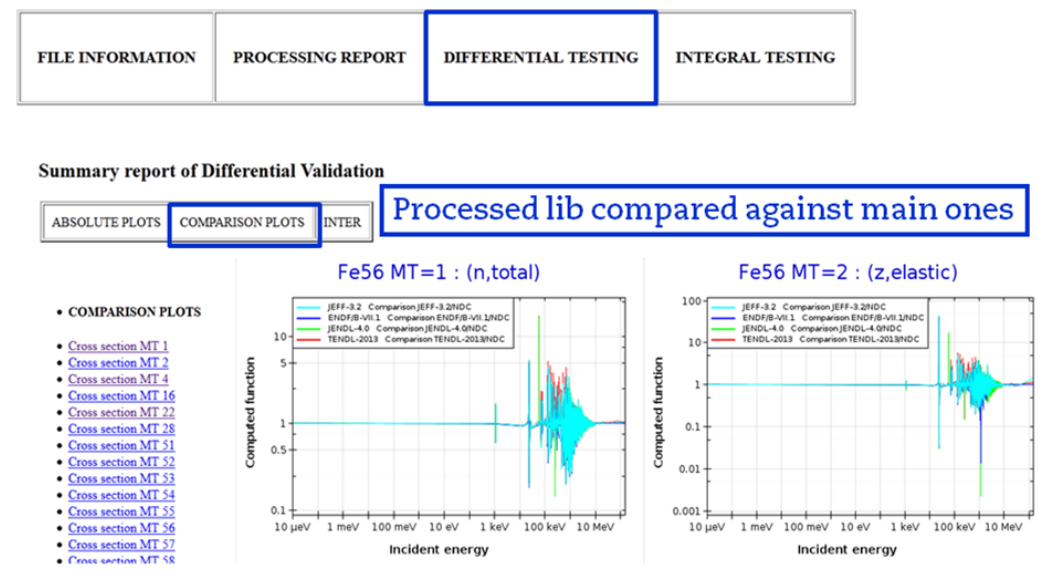

Figure 2. Screen-shot of the 'processing' panel of the NDEC prototype showing the visual comparison of ratio of processed cross sections with major nuclear data library releases (ENDF/B-VII.1; JENDL-4.0; TENDL-2013; JEFF-3.2).

A large computation and compilation effort of criticality sensitivity profiles for available ICSBEP[3] and IRPHE[4] benchmark experiments has been undertaken at the NEA in recent years, resulting in the creation of software searching tools that are able to interrogate the integral databases and select the pertinent benchmark cases (nuclide/reaction specific, above a given sensitivity threshold to $k_{e f f}$ ); these are the DICE (Database for the International Criticality Safety Benchmark Evaluation Project) or the IDAT (International Reactor Physics Handbook Database and Analysis Tool) tools. A 


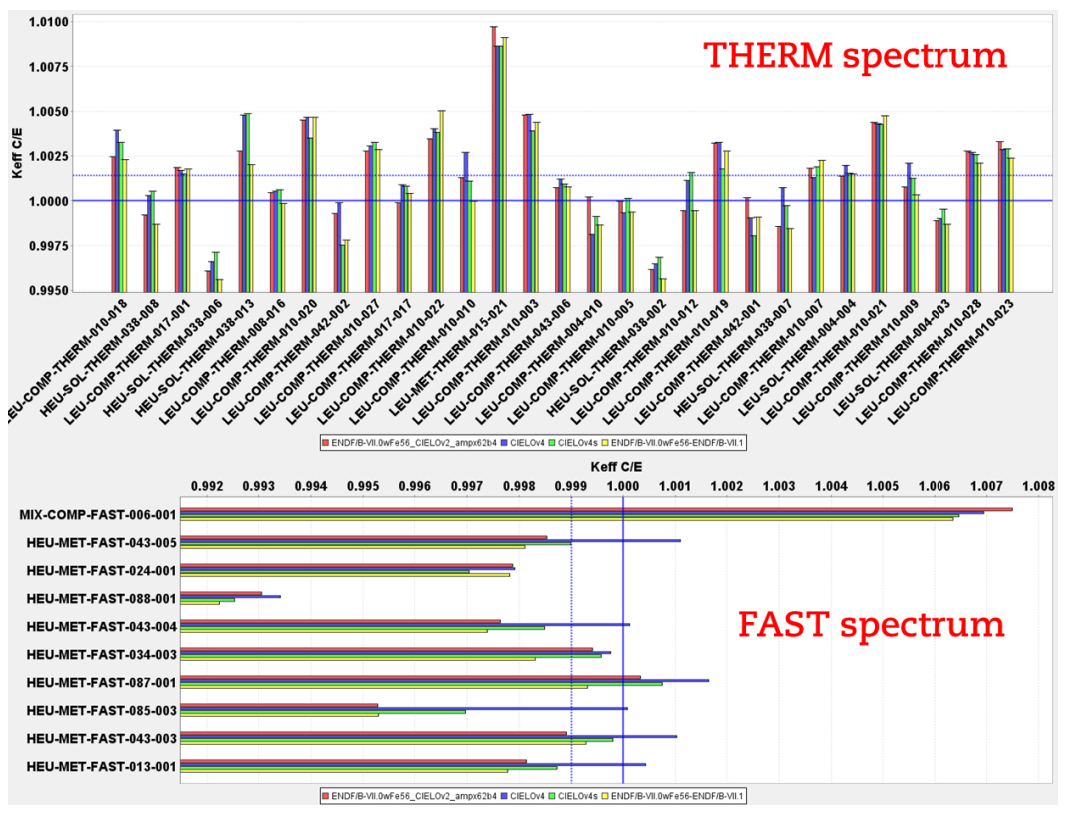

Figure 3. Screen-shot showing criticality benchmark results for 4 different Fe-56 evaluations being considered in the WPEC/CIELO initiative and tested with NDEC.

new tool called NDaST (Nuclear Data Sensitivity Tool)[5] has been recently developed ${ }^{1}$ to make use of this sensitivity profile information and carry out sensitivity-based calculations; either performing broad perturbations to nuclear data as a function of nuclide, reaction and energy range or, through an interface JANIS, perform uncertainty propagation of nuclear covariance data to the given response parameter (e.g. keff). The calculated results of both methods allow the quantitative analysis of the impact upon a large number of selected benchmark cases and can be used to construct the pertinent benchmark validation suites per nuclide and application tested (see Figure 3).

\subsection{Foreseen developments}

The development of NDEC is still ongoing, and many developments could still be envisaged in the medium to longer term. Concerning integral validation, the enlarged scope beyond criticality benchmarking should be considered and could include the selection of relevant decay cases (decay heat, doses, reaction rates), the comparison to spectral average cross sections included in EXFOR, inclusion of transmission and shielding benchmarks from ICSBEP and SINBAD databases, or even performing simple computational benchmarks between evaluated files (e.g. critical mass, shielding transmission, particle emission, etc.) among others.

\section{Conclusions}

Centralization of processes and tools that allow for a thorough assessment of evaluated nuclear data and its performance in integral experiments is seen as a key necessity of modern nuclear data QA.

\footnotetext{
${ }^{1} \mathrm{NDaST}$ is freely available online via the NEA webpage (http://www.oecd-nea.org/ndast/).
} 
The development of the NDEC platform is at the center of the initiative undertaken by the NEA Data Bank to strengthen its role as a reference centre in verification, benchmarking and validation of nuclear data. These efforts aim at providing better nuclear data services to the Data Bank's member countries in general and to serve the Joint Evaluated Fission and Fusion (JEFF) Nuclear Data Library project in particular.

\section{References}

[1] C.J. Diez, F. Michel-Sendis, O. Cabellos, On the processing of JEFF-3.2 neutron data library with AMPX 6.2 for its use with the SCALE tool suite, these proceedings.

[2] N. Soppera, M. Bossant, E. Dupont, "JANIS 4: An Improved Version of the NEA Java-based Nuclear Data Information

[3] OECD NEA, International Handbook of Evaluated Criticality Safety Benchmark Experiments, NEA/NSC/DOC(95)03, OECD. And at www.oecd-nea.org/science/wpncs/icsbep/

[4] OECD NEA, International Handbook of Evaluated Reactor Physics Benchmark Experiments, NEA/NSC/DOC(2006)1, OECD. And at www.oecd-nea.org/science/wprs/irphe

[5] J. Dyrda, I. Hill, et al, The New OECD-NEA Nuclear Data and Sensitivity Tool (NDaST), ANS Winter meeting proceedings, Washington DC, USA, Nov.8-12, 2015. 
